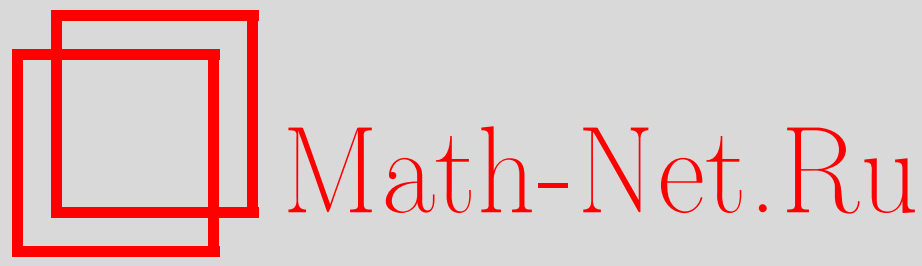

В. И. Алхимов, Канонический ансамбль частиц, блуждающих без самопересечений, ТМФ, 2017, том 191, номep 1, 100-115

DOI: https://doi.org/10.4213/tmf9154

Использование Общероссийского математического портала Math-Net.Ru подразумевает, что вы прочитали и согласны с пользовательским соглашением http: //www . mathnet.ru/rus/agreement

Параметры загрузки:

IP : 54.162 .127 .20

26 апреля 2023 г., 13:34:47

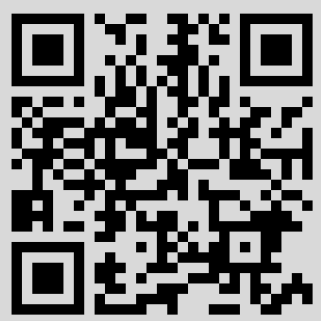




\title{
ФИЗИКА
}

Том 191, № 1

апрель, 2017

(C) 2017 г.

\author{
В. И. Алхимов*
}

\section{КАНОНИЧЕСКИЙ АНСАМБЛЬ ЧАСТИЦ, БЛУЖДАЮЩИХ БЕЗ САМОПЕРЕСЕЧЕНИЙ}

\begin{abstract}
Рассматривается ансамбль не взаимодействующих между собой частиц, случайно блуждающих в $d$-мерном евклидовом пространстве $\mathbb{R}^{d}$. Отдельные перемещения каждой частицы подчинены одному и тому же распределению, но после завершения каждого такого перемещения ее положение фиксируется "меткой" - областью в виде шара диаметра $r_{0}$, недоступной для последующих посещений этой частицей. В результате в пространстве $\mathbb{R}^{d}$ получен соответствующий ансамбль "меченых" траекторий, в каждой из которых расстояние между центрами любой пары указанных шаров больше $r_{0}$. Изложен метод вычисления асимптотики плотности вероятности $W_{n}(\mathbf{r})$ расстояния $r$ между центрами начального и конечного шаров траектории, состоящей из $n$ отдельных перемещений некоторой частицы ансамбля. Помимо расстояния $r$, случайной величиной в рассматриваемой модели является и число $n$ - модуль траектории, что обусловливает необходимость определения распределения для $n$, в качестве которого используется каноническое распределение, полученное при наиболее вероятном распределении частиц в ансамбле по модулям их траекторий. Усреднение плотности $W_{n}(\mathbf{r})$ по каноническому распределению модуля $n$ позволяет найти асимптотику плотности вероятности расстояния $r$ между концами траекторий частиц канонического ансамбля, блуждающих без самопересечений в $\mathbb{R}^{d}$, когда $2 \leqslant d<4$.
\end{abstract}

Ключевые слова: канонический ансамбль, блуждания без самопересечений, основное уравнение, ренормализационная группа, метод перевала, асимптотическое распределение.

DOI: https://doi.org/10.4213/tmf9154

\section{1. ВВЕДЕНИЕ}

В данной работе рассматривается ансамбль частиц, блуждающих в $d$-мерном евклидовом пространстве $\mathbb{R}^{d}$, заполненном однородной изотропной физической средой. Отдельные перемещения $\mathbf{r}_{k}, k=1,2, \ldots$, каждой частицы подчинены одному и тому же распределению плотности вероятности $w\left(\mathbf{r}_{k}\right)$ со средним квадратом $\left\langle r^{2}\right\rangle$ отдельного перемещения. Кроме того, после завершения каждого такого перемещения частицы ее положение в среде фиксируется "меткой" - областью в виде шара

*Факультет информационных технологий, Московский государственный психолого-педагогический университет, Москва, Россия. E-mail: alvaliv@list.ru 
диаметра $r_{0}$, которая является недоступной для последующих посещений этой частицей. В результате указанного ограничения на положения частиц в $\mathbb{R}^{d}$ мы получаем соответствующий ансамбль “меченых" траекторий, в каждой из которых расстояние между центрами любой пары указанных шаров больше $r_{0}$. Обозначим через $\mathbf{r}_{k}$ вектор, соединяющий центры $(k-1)$-го и $k$-го шаров, $\mathbf{r}_{k l}-$ вектор, соединяющий центры $(k-1)$-го и $l$-го шаров, где $k \leqslant l$. Тогда $r_{1 n} \equiv\left|\mathbf{r}_{1 n}\right|$ обозначает расстояние между центрами начального (0-го) и конечного ( $n$-го) шаров траектории, состоящей из $n$ отдельных перемещений частицы ансамбля. Таким образом, расстояние $r_{1 n}$ и число $n$, называемое в дальнейшем модулем траектории, являются ее основными характеристиками. Введенный запрет на пересечение шаров, принадлежащих траектории какой-либо частицы, оказывает существенное влияние на функциональную зависимость плотности вероятности $W_{n}(\mathbf{r})$ от расстояния $r=\left|\mathbf{r}_{1 n}\right|$ между концами этой траектории. Помимо расстояния $r$, случайной величиной в рассматриваемой модели является модуль $n$ траектории, что обусловливает необходимость определения распределения для $n$.

Предположим, что число $M$ частиц в ансамбле равно сумме $m_{0}+m_{1}+m_{2}+\cdots$, где $m_{0}$ - число частиц, не имеющих перемещений, $m_{1}$ - число частиц, траектории которых состоят из одного модуля, $m_{2}$ - число частиц, траектории которых состоят из двух модулей, и т. д. Тогда посредством набора целых чисел $\left\{m_{n}\right\}$ описывается любое распределение частиц по модулям их траекторий. Далее допустим, что кроме числа $M$ задано среднее значение $N$ модуля по траекториям всех частиц, т. е.

$$
\sum_{n=0}^{\infty} m_{n}=M, \quad \sum_{n=0}^{\infty} n m_{n}=M N .
$$

При выполнении условий (1) и $M \rightarrow \infty$ с помощью метода Дарвина-Фаулера можно получить наиболее вероятный набор $\left\{\bar{m}_{n}\right\}$, определяющий каноническое распределение [1]-[3]. Если доказать, что $\left\{m_{n}\right\} \sim\left\{\bar{m}_{n}\right\}$ при $M \rightarrow \infty$, то указанное распределение находится путем вычисления среднего значения $\left\langle m_{n}\right\rangle$ по всем возможным распределениям модулей. В результате при $M \rightarrow \infty$ для значений $\left\langle m_{k}\right\rangle$ и дисперсии $\mathbb{D} m_{k} \equiv\left\langle m_{k}^{2}\right\rangle-\left\langle m_{k}\right\rangle^{2}$ имеем

$$
\begin{aligned}
& \lim _{M \rightarrow \infty} \frac{\left\langle m_{k}\right\rangle}{M} \equiv \mathbb{P}(n)=(1-q) q^{n}, \\
& \lim _{M \rightarrow \infty} \mathbb{D} m_{k}=0 .
\end{aligned}
$$

Следовательно, $\left\{m_{n}\right\} \sim\left\{\bar{m}_{n}\right\}$ и, таким образом, формула (2), называемая геометрическим распределением, является точной, а величина $q$ определяется равенством

$$
N=\mathrm{E} n \equiv \sum_{n=0}^{\infty} n \mathbb{P}(n)
$$

из которого находим $q=N(N+1)^{-1}$. Таким образом, формула (2) определяет искомую вероятность найти траекторию частицы с модулем $n$ при наиболее вероятном распределении частиц в ансамбле по модулям их траекторий. Если теперь 
плотность вероятности $W_{n}(\mathbf{r})$ усреднить по указанному в $(2)$ распределению $\mathbb{P}(n)$, то получим

$$
\mathrm{E} W_{n}(\mathbf{r})=(1-q) W(q, \mathbf{r})
$$

где

$$
W(z, \mathbf{r}) \equiv \sum_{n=0}^{\infty} z^{n} W_{n}(\mathbf{r})
$$

- ее производящая функция.

\section{2. ОСНОВНОЕ УРАВНЕНИЕ И РЕНОРМГРУППОВАЯ ИНВАРИАНТНОСТЬ}

Искомая плотность вероятности $W_{n}(\mathbf{r})$ для рассматриваемой здесь модели определяется равенством

$$
W_{n}(\mathbf{r})=Q_{n}^{-1} \int \delta\left(\mathbf{r}-\sum_{k=1}^{n} \mathbf{r}_{k}\right) P_{1 n} \prod_{k=1}^{n} w\left(\mathbf{r}_{k}\right) d \mathbf{r}_{k}
$$

где $\delta(\mathbf{r})$ - дельта-функция Дирака, $Q_{n}$ - нормировочный множитель, равный

$$
\begin{aligned}
Q_{n} & =\int P_{1 n} \prod_{k=1}^{n} w\left(\mathbf{r}_{k}\right) d \mathbf{r}_{k}, \\
P_{1 n} & =\prod_{1 \leqslant j \leqslant k \leqslant n}\left(1-\theta_{j k}\right), \quad \theta_{j k} \equiv \theta\left(r_{0}-r_{j k}\right),
\end{aligned}
$$

$\theta(x)$ - функция Хевисайда, $d \mathbf{r}$ - элемент объема, который в гиперсферических полярных координатах пространства $\mathbb{R}^{d}$ принимает вид $d \mathbf{r}=r^{2 s+1} d r d \omega, s=(d-2) / 2$, $d \omega$ - элемент поверхности сферы единичного радиуса, $\omega=2 \pi^{s+1} / \Gamma(s+1)$ - полная поверхность этой сферы, а $\Gamma(x)$ - гамма-функция Эйлера. Наконец, мы полагаем, что плотность вероятности $w(\mathbf{r})$ зависит лишь от длины $r$ отдельного перемещения частицы, сосредоточена и непрерывна на интервале $\left(r_{0}, \infty\right)$ и достаточно быстро убывает при $r \rightarrow \infty$. Используя фурье-представление дельта-функции, запишем плотность $W_{n}(\mathbf{r})$ в форме интеграла Фурье:

$$
W_{n}(\mathbf{r})=(2 \pi)^{-d} \int_{\mathbb{R}^{d}} e^{-i \mathbf{r} \cdot \mathbf{p}} \widetilde{W}_{n}(\mathbf{p}) d \mathbf{p}
$$

где характеристическая функция $\widetilde{W}_{n}(\mathbf{p})$ плотности $W_{n}(\mathbf{r})$ определяется равенством

$$
\widetilde{W}_{n}(\mathbf{p})=Q_{n}^{-1} \int P_{1 n} \prod_{k=1}^{n} e^{i \mathbf{p} \cdot \mathbf{r}_{k}} w\left(\mathbf{r}_{k}\right) d \mathbf{r}_{k} .
$$

Однако для решения рассматриваемой задачи более удобно использовать ненормированную функцию

$$
V_{n}(\mathbf{r})=Q_{n} W_{n}(\mathbf{r})
$$

и ее фурье-образ

$$
\widetilde{V}_{n}(\mathbf{p})=Q_{n} \widetilde{W}_{n}(\mathbf{p})
$$


В работе [4] для производящей функции

$$
\widetilde{V}(z, \mathbf{p}) \equiv \sum_{n=0}^{\infty} z^{n} \widetilde{V}_{n}(\mathbf{p})
$$

установлено уравнение

$$
\widetilde{V}^{-1}(z, \mathbf{p})=\widetilde{V}_{0}^{-1}(z, \mathbf{p})-f(z, \mathbf{p})
$$

при условии

$$
|z \tilde{w}(\mathbf{p})+f(z, \mathbf{p})|<1
$$

где

$$
\begin{aligned}
\widetilde{V}_{0}^{-1}(z, \mathbf{p}) & =1-z \tilde{w}(\mathbf{p}) \\
\tilde{w}(\mathbf{p}) \equiv \int e^{i \mathbf{p} \cdot \mathbf{r}} w(\mathbf{r}) d \mathbf{r} & =\omega \int_{0}^{\infty} \Lambda_{s}(p r) w(r) r^{2 s+1} d r
\end{aligned}
$$

- характеристическая функция плотности $w(\mathbf{r}), \Lambda_{s}(z) \equiv \Gamma(s+1)(2 / z)^{s} J_{s}(z), J_{s}(z)-$ функция Бесселя первого рода, а функция $f(z, \mathbf{p})$ определена выражением

$$
f(z, \mathbf{p})=-\frac{1}{(2 \pi)^{d}} \int d \mathbf{p}_{1} \tilde{\theta}\left(\mathbf{p}_{1}\right) \tilde{V}\left(z, \mathbf{p}-\mathbf{p}_{1}\right) Y\left(z, \mathbf{p}, \mathbf{p}_{1}, \mathbf{p}-\mathbf{p}_{1}\right),
$$

в котором так называемая "вершинная" функция $Y\left(z, \mathbf{p}, \mathbf{p}_{1}, \mathbf{p}-\mathbf{p}_{1}\right)$ задана посредством ряда:

$$
\begin{aligned}
Y(z, & \left.\mathbf{p}, \mathbf{p}_{1}, \mathbf{p}-\mathbf{p}_{1}\right) \equiv 1-\frac{1}{(2 \pi)^{d}} \int d \mathbf{p}_{2} \tilde{\theta}\left(\mathbf{p}_{2}\right) \tilde{V}\left(z, \mathbf{p}-\mathbf{p}_{1}-\mathbf{p}_{2}\right) \tilde{V}\left(z, \mathbf{p}-\mathbf{p}_{2}\right)+ \\
& +\frac{1}{(2 \pi)^{2 d}} \iint d \mathbf{p}_{2} d \mathbf{p}_{3} \tilde{\theta}\left(\mathbf{p}_{2}\right) \tilde{\theta}\left(\mathbf{p}_{3}\right) \tilde{V}\left(z, \mathbf{p}-\mathbf{p}_{1}-\mathbf{p}_{2}\right) \times \\
& \times\left\{\widetilde{V}\left(z, \mathbf{p}-\mathbf{p}_{2}\right) \widetilde{V}\left(z, \mathbf{p}-\mathbf{p}_{2}-\mathbf{p}_{3}\right) \tilde{V}\left(z, \mathbf{p}-\mathbf{p}_{3}\right)+\right. \\
& +\widetilde{V}\left(z, \mathbf{p}-\mathbf{p}_{1}-\mathbf{p}_{2}-\mathbf{p}_{3}\right) \widetilde{V}\left(z, \mathbf{p}-\mathbf{p}_{1}-\mathbf{p}_{3}\right) \widetilde{V}\left(z, \mathbf{p}-\mathbf{p}_{3}\right)+ \\
& +\widetilde{V}\left(z, \mathbf{p}-\mathbf{p}_{1}-\mathbf{p}_{2}-\mathbf{p}_{3}\right) \widetilde{V}\left(z, \mathbf{p}-\mathbf{p}_{2}-\mathbf{p}_{3}\right) \widetilde{V}\left(z, \mathbf{p}-\mathbf{p}_{2}\right)+ \\
& \left.+\widetilde{V}\left(z, \mathbf{p}-\mathbf{p}_{1}-\mathbf{p}_{2}-\mathbf{p}_{3}\right) \widetilde{V}\left(z, \mathbf{p}-\mathbf{p}_{2}-\mathbf{p}_{3}\right) \widetilde{V}\left(z, \mathbf{p}-\mathbf{p}_{3}\right)\right\}-\cdots,
\end{aligned}
$$

здесь фурье-образ $\tilde{\theta}(\mathbf{p})$ функции $\theta\left(r_{0}-r\right)$ определяется равенством

$$
\tilde{\theta}(\mathbf{p})=\int_{\mathbb{R}^{d}} e^{i \mathbf{p} \cdot \mathbf{r}} \theta\left(r_{0}-r\right) d \mathbf{r}=v \Lambda_{s+1}\left(r_{0} p\right),
$$

в котором $v=\Gamma^{-1}(s+2)\left(\pi r_{0}^{2}\right)^{s+1}$ обозначает объем шара диаметра $r_{0}$. Уравнение $(7)$ по своей форме аналогично известному уравнению Дайсона в квантовой теории поля и в рассматриваемой задаче играет основополагающую роль, в связи с чем мы будем называть его основным уравнением. Особым свойством ряда в равенстве (12) является тот факт, что при переходе от одних членов ряда к другим появление под знаком интеграла величины $\{-\tilde{\theta}\}$ всегда сопровождается умножением ее на произведение $\{\widetilde{V}\}^{2}$ двух искомых функций. Это свойство можно продемонстрировать, представив ряд в (12) в символической форме:

$$
Y(z, \mathbf{p})=\sum_{n \geqslant 0} \int\left\{\frac{d \mathbf{p}^{\prime}}{(2 \pi)^{d}}\right\}^{n}\left\{-\tilde{\theta}\left(\mathbf{p}^{\prime}\right)\right\}^{n}\left\{\tilde{V}\left(z, \mathbf{p}-\mathbf{p}^{\prime}\right)\right\}^{2 n} .
$$


Отсюда вытекает важное свойство основного уравнения (7) - его инвариантность относительно мультипликативных преобразований:

$$
\begin{gathered}
\tilde{V} \rightarrow \widetilde{V}^{\prime}=\alpha \tilde{V}, \quad \widetilde{V}_{0} \rightarrow \widetilde{V}_{0}^{\prime}=\alpha \widetilde{V}_{0}, \quad \tilde{\theta} \rightarrow \tilde{\theta}^{\prime}=\alpha^{-2} \tilde{\theta}, \\
f \rightarrow f^{\prime}=\alpha^{-1} f, \quad Y \rightarrow Y^{\prime}=Y,
\end{gathered}
$$

где $\alpha$ - не равный нулю непрерывный параметр. Эти преобразования образуют непрерывную группу, называемую обычно ренормгруппой (РГ), в связи с чем свойство (14) является исходной точкой для применения метода РГ в исследуемой здесь задаче. Формулы (11) и (12) описывают искомую связь между функциями $f(z, \mathbf{p})$ и $\widetilde{V}(z, \mathbf{p})$, составляя вместе с основным уравнением (7) замкнутую систему при условии сходимости ряда в (12). Это условие следует из выражения (6), если в нем учесть неравенство $\left|\widetilde{V}_{n}(\mathbf{p})\right| \leqslant 1$, вытекающее из формул (4) и (5). В результате находим

$$
|\widetilde{V}(z, \mathbf{p})| \leqslant(1-|z|)^{-1}, \quad|z|<1,
$$

и, следовательно, ряд в правой части равенства (6) абсолютно и равномерно сходится для всех вещественных значений $p$ и $|z|<1$. Благодаря этим условиям и ограниченности функции $|\tilde{w}(\mathbf{p})|$ можно утверждать согласно неравенству (8), что функция $f(z, \mathbf{p})$ и, следовательно, правая часть равенства (11) ограничены по модулю, что обусловливает сходимость ряда в (12). Если бы удалось отыскать решение уравнения (7), то с помощью формул обращения

$$
\begin{aligned}
W_{n}(\mathbf{r}) & =\left[\oint_{\gamma} \frac{d z}{z^{n+1}} \tilde{V}(z, 0)\right]^{-1} \oint_{\gamma} \frac{d z}{z^{n+1}} V(z, \mathbf{r}), \\
V(z, \mathbf{r}) & =(2 \pi)^{-d} \int_{\mathbb{R}^{d}} e^{-i \mathbf{r} \cdot \mathbf{p}} \widetilde{V}(z, \mathbf{p}) d \mathbf{p}
\end{aligned}
$$

мы пришли бы к искомому результату.

Однако проблема решения основного уравнения и последующего определения функции $W_{n}(\mathbf{r})$ чрезвычайно сложна. Более реальной задачей в этом случае является вычисление асимптотики плотности $W_{n}(\mathbf{r})$ при больших значениях $n$ и $r$. Но согласно формулам (15) и (16) для этого необходимо знать поведение функции $\widetilde{V}(z, \mathbf{p})$ при малых значениях $\left|z-z_{0}\right|$ и $p$, где $z_{0}$ - ближайшая к началу координат $z=0$ особая точка функции

$$
\widetilde{V}(z, 0)=Q(z) \equiv \sum_{n=0}^{\infty} z^{n} Q_{n},
$$

т. е. такая точка, которая удовлетворяет тождеству

$$
1-z_{0}-f\left(z_{0}, 0\right) \equiv 0
$$

в соответствии с соотношениями (5)-(9). Очевидно, что величина $0<z_{0} \leqslant 1$ зависит от объема $v$ "метки", который приводит к смещению особой точки $z_{0}=z_{0}(v)$ из ее "невозмущенного" положения $z_{0}(0)=1$. В теории аналитических функций утверждается, что граница круга сходимости степенного ряда проходит через ближайшую к началу координат его особую точку. Кроме того, асимптотическое поведение функции, представленной степенным рядом, при приближении переменной $z$ 
к границе круга сходимости можно связать с асимптотическим поведением коэффициентов этого ряда, когда их порядковый номер стремится к бесконечности [5]. Действительно, известная тауберова теорема в приложении к степенному ряду (17) утверждает, что вследствие условия $z_{0} Q_{n+1} / Q_{n}<1$ последовательность коэффициентов $Q_{n} z_{0}^{n}$ ряда

$$
Q(z)=\sum_{n=0}^{\infty} Q_{n} z_{0}^{n}\left(\frac{z}{z_{0}}\right)^{n}, \quad\left|\frac{z}{z_{0}}\right|<1,
$$

монотонна и при $0 \leqslant \alpha<\infty$ соотношения

$$
Q(z) \sim\left(1-\frac{z}{z_{0}}\right)^{-\alpha} C\left(\frac{z_{0}}{z_{0}-z}\right), \quad \frac{z}{z_{0}} \rightarrow 1-,
$$

и

$$
Q_{n} \sim \frac{n^{\alpha-1}}{\Gamma(\alpha) z_{0}^{n}} C(n), \quad n \rightarrow \infty,
$$

равносильны, где $C(t)$ - медленно меняющаяся на бесконечности положительная функция [6]. Важность тауберовой теоремы здесь обусловлена ее тесной связью с проблемой вычисления асимптотики плотности вероятности $W_{n}(r)$, когда $n$ и $r$ стремятся к бесконечности. Далее пусть $p_{ \pm} \equiv \pm i \eta-$ ближайшие к началу $p=0$ корни уравнения $\widetilde{V}^{-1}(z, \mathbf{p})=0$, где $z$ принадлежат окрестности точки $z_{0}$. Тогда, полагая $p=i \eta$ в уравнении (7), получим тождество

$$
1-z \tilde{w}(i \eta)-f(z, i \eta) \equiv 0,
$$

с помощью которого уравнение (7) преобразуется к виду

$$
\widetilde{V}^{-1}(z, p)=z(\tilde{w}(i \eta)-\tilde{w}(p))+f(z, i \eta)-f(z, p) .
$$

Теперь обе части уравнения (20) умножим на новую переменную $\zeta=z_{0} / z$ и введем обозначения

$$
\begin{gathered}
\bar{V}(\zeta, p)=\zeta^{-1} \tilde{V}(z, p), \quad \bar{V}_{0}^{-1}(\zeta, p)=z_{0}(\tilde{w}(i \eta)-\tilde{w}(p)) \\
F(\zeta, p ; \bar{\theta} ; \bar{V})=\zeta f(z, p), \quad \bar{\theta}=\zeta^{2} \tilde{\theta}, \quad \bar{v}=\zeta^{2} v
\end{gathered}
$$

В результате приходим к соотношению

$$
\bar{V}^{-1}(\zeta, p)=\bar{V}_{0}^{-1}(\zeta, p)+F(\zeta, i \eta ; \bar{\theta} ; \bar{V})-F(\zeta, p ; \bar{\theta} ; \bar{V})
$$

в котором функцию $\bar{V}_{0}^{-1}(\zeta, p)$ можно записать с помощью (10) в виде ряда

$$
\bar{V}_{0}^{-1}(\zeta, p)=z_{0} \sum_{m=1}^{\infty} \frac{\Gamma(1+s)}{4^{m} m ! \Gamma(1+s+m)}\left[\eta^{2 m}+(-1)^{m+1} p^{2 m}\right]\left\langle r^{2 m}\right\rangle,
$$

где $\left\langle r^{2 m}\right\rangle$ - моменты $2 m$-го порядка случайной величины $r$. Отсюда для малых значений $|\eta|$ и $|p|$ имеем

$$
\bar{V}_{0}^{-1}(\zeta, p) \simeq \frac{z_{0}\left\langle r^{2}\right\rangle}{2 d}\left(\eta^{2}+p^{2}\right)
$$


Кроме того, из тождеств (18) и (19) следует формула

$$
\zeta-1=\bar{V}_{0}^{-1}(\zeta, 0)+F(\zeta, i \eta ; \bar{\theta} ; \bar{V})-F(1,0 ; \bar{\theta} ; \bar{V})
$$

устанавливающая функциональную зависимость $\eta=\eta(\zeta)$, согласно которой $\eta(1)=0$. Уравнение (21) совместно с равенством (24) составляют основу излагаемого здесь метода исследования данной проблемы. В новых переменных формулы (15) и (16) теперь принимают следующий вид:

$$
W_{n}(\mathbf{r})=\left[\int_{\bar{\gamma}} \zeta^{n} \bar{V}(\zeta, 0) d \zeta\right]^{-1} \int_{\bar{\gamma}} \zeta^{n} \widehat{V}(\zeta, \mathbf{r}) d \zeta
$$

где

$$
\widehat{V}(\zeta, \mathbf{r})=(2 \pi)^{-d} \int_{\mathbb{R}^{d}} e^{-i \mathbf{r} \cdot \mathbf{p}} \bar{V}(\zeta, \mathbf{p}) d \mathbf{p},
$$

а контур интегрирования $\bar{\gamma}$ в $(25)$ расположен в комплексной плоскости переменной $\zeta$ справа от единичного круга, на границе которого в точке $\zeta=1$ и внутри него расположены все особые точки подынтегральной функции.

Описанные выше свойства основного уравнения служат базой для решения его с помощью метода РГ, суть которого состоит в следующем. Обратимся к уравнениям $(21)$ и $(24)$, первое из которых определяет тип особых точек $p_{ \pm} \equiv \pm i \eta$ функции $\bar{V}(\zeta, p)$, а второе - зависимость между величинами $\zeta$ и $\eta$. Удобнее начать с определения характера сингулярности функции $\bar{V}(\zeta, p)$ в указанных особых точках. С этой целью запишем искомую функцию $\bar{V}(\zeta, p)$ в виде

$$
\bar{V}(\zeta, p)=\bar{V}_{0}(\zeta, p) G,
$$

где величина $G$ согласно (21) и (27) удовлетворяет уравнению

$$
G^{-1}=1+\Phi\left(\eta^{2}, \rho^{2} ; \bar{\theta} ; G\right)
$$

в котором введены новые переменные $\eta^{2}$ и $\rho^{2} \equiv \eta^{2}+p^{2}$, а также новая функция

$$
\Phi\left(\eta^{2}, \rho^{2} ; \bar{\theta} ; G\right)=\bar{V}_{0}(\zeta, p)\left[F\left(\zeta, i \eta ; \bar{\theta} ; \bar{V}_{0} G\right)-F\left(\zeta, p ; \bar{\theta} ; \bar{V}_{0} G\right)\right] .
$$

Теперь предположим, что при некотором значении $\rho^{2}=\lambda$ имеет место равенство $\Phi\left(\eta^{2}, \lambda ; \bar{\theta} ; G\right)=0$, т. е. для функции $G$ выполняется условие нормировки $\left.G\right|_{\rho^{2}=\lambda}=1$. Тогда РГ-свойство уравнения (14) в обозначениях $x=\eta^{2} / \lambda, y=\rho^{2} / \lambda$ можно записать в виде

$$
G(x, y ; \bar{\theta})=G(x, t ; \bar{\theta}) G\left(\frac{x}{t}, \frac{y}{t} ; \bar{\theta} G^{2}(x, t ; \bar{\theta})\right),
$$

где $t$ - отличный от нуля непрерывный параметр. Возведя обе части последнего равенства в квадрат и затем умножив на $\bar{\theta}$, мы получим для инвариантной относительно РГ-преобразований величины

$$
B(x, y ; \bar{\theta})=\bar{\theta} G^{2}(x, y ; \bar{\theta})
$$

функциональное уравнение

$$
B(x, y ; \bar{\theta})=B\left(\frac{x}{t}, \frac{y}{t} ; B(x, t ; \bar{\theta})\right)
$$


со следующим условием нормировки:

$$
B(x, 1 ; \bar{\theta})=\bar{\theta}
$$

Уравнение (30) является замкнутым и может быть решено в общем виде [7], [8]. Однако для практических целей более удобно иметь дело с дифференциальными уравнениями Ли в силу непрерывности РГ. Чтобы получить уравнение Ли, например, для $B(x, y ; \bar{\theta})$, продифференцируем обе части равенства (30) по у и затем положим $t=y$. В результате имеем

$$
y \frac{\partial B(x, y ; \bar{\theta})}{\partial y}=\beta\left(\frac{x}{y} ; B(x, y ; \bar{\theta})\right),
$$

где

$$
\beta(x, \bar{\theta})=\left.\frac{\partial B(x, y ; \bar{\theta})}{\partial y}\right|_{y=1},
$$

а граничным условием служит условие нормировки (31). Приведенные выше РГуравнения оказываются особенно полезными при исследовании асимптотических свойств функции $G(x, y ; \bar{\theta})$. Например, чтобы определить поведение функции $G(x, y ; \bar{\theta})$, когда $x \ll y$ и $y \rightarrow 0$, выберем точку нормировки $\lambda$, удовлетворяющую неравенству $\eta^{2} \ll \rho^{2}<\lambda$, и в уравнении (32) перейдем к пределу, устремляя $\eta^{2}$ к нулю. Тогда, обозначая

$$
\begin{aligned}
B(y ; \bar{\theta}) & =\lim _{x \rightarrow 0} B(x, y ; \bar{\theta}), \\
\beta(\bar{\theta}) & =\left.\frac{\partial B(y ; \bar{\theta}}{\partial y}\right|_{y=1},
\end{aligned}
$$

мы придем к следующему уравнению:

$$
y \frac{\partial B(y ; \bar{\theta})}{\partial y}=\beta(B(y ; \bar{\theta}))
$$

с граничным условием

$$
B(1 ; \bar{\theta})=\bar{\theta}
$$

Уравнение (34) совместно с равенством (35) можно записать в форме уравнения Гелл-Манна-Лоу:

$$
\int_{\bar{\theta}}^{B(y ; \bar{\theta})} \beta^{-1}(t) d t=\ln y,
$$

очень удобной для анализа поведения инвариантного объема $B(y ; \bar{\theta})$ в области малых значений $y$ или $\rho^{2} \ll \lambda[9]$. Таким образом, согласно РГ-уравнениям, эффективным параметром, характеризующим интенсивность объемного взаимодействия в малой окрестности точек $\eta=0$ и $\rho=0$, является инвариантный объем. Поэтому, чтобы определить асимптотику, например, функции $G(y ; \bar{\theta})$ при $y \rightarrow 0$, необходимо знать в этом случае поведение величины $B(y ; \bar{\theta})$, которое, в свою очередь, определяется свойствами функции $\beta(B)$ в соответствии с (33). Поскольку для вычисления функции $\beta(B)$ обычно используется теория возмущений, то реально можно судить о ее поведении лишь в малой окрестности точки $B=0$, в которой $\beta(0)=0$. Действительно, если в этой окрестности величина $\beta(B)$ положительна, то инвариантный объем $B(y ; \bar{\theta})$ стремится к нулю, когда $y \rightarrow 0$. Если же функция $\beta(B)$ отрицательна вблизи нуля, то при $y \rightarrow 0$ величина $B(y ; \bar{\theta})$ возрастает, в результате чего мы выходим за рамки применимости теории возмущений. 


\section{3. АСИМПТОТИКА ПЛОТНОСТИ ВЕРОЯТНОСТИ}

Обратимся к уравнению (21) и рассмотрим функцию $F(\zeta, p ; \bar{\theta} ; \bar{V})$ в его правой части, определяемую посредством ряда согласно выражениям (11) и (12). Переход от одного члена этого ряда к следующему сопровождается появлением интеграла, который на примере перехода от первого члена ряда ко второму имеет вид

$$
I\left(\mathbf{p}, \mathbf{p}_{1}\right)=-\int \frac{d \mathbf{p}_{2}}{(2 \pi)^{d}} \bar{\theta}\left(\mathbf{p}_{2}\right) \bar{V}\left(\zeta, \mathbf{p}-\mathbf{p}_{1}-\mathbf{p}_{2}\right) \bar{V}\left(\zeta, \mathbf{p}-\mathbf{p}_{2}\right),
$$

что свидетельствует о РГ-инвариантности основного уравнения. Если теперь под знаком интеграла в $(36)$ функцию $\bar{V}$ заменить на $\bar{V}_{0}$, т. е. использовать "нулевое" приближение, то, учитывая (13) и (22), после замены переменных $p=\eta p^{\prime}, p_{1}=\eta p_{1}^{\prime}$, $p_{2}=\eta p_{2}^{\prime}$ мы получим, что $I\left(\mathbf{p}, \mathbf{p}_{1}\right)=O\left(\eta^{2(s-1)}\right)$, когда $\eta \rightarrow 0$. Это означает, что в случае $s \leqslant 1(d \leqslant 4)$ рассматриваемое ограничение на самопересечения траектории оказывает существенное влияние на искомый вид асимптотики плотности вероятности $W_{n}(\mathbf{r})$. Поскольку в окрестности точек $\eta=0$ и $p=0$ переменные $\eta$ и $p$ присутствуют в (21) главным образом в виде комбинации $\eta^{2}+p^{2} \equiv \rho^{2}$, то для значений $s \leqslant 1(d \leqslant 4)$ величина $\Phi\left(\eta^{2}, \rho^{2} ; \bar{\theta} ; G\right)$ как функция от $\rho$ должна неограниченно возрастать при $\rho \rightarrow 0$. Поэтому точки $p_{ \pm} \equiv \pm i \eta$ не являются простыми нулями функции $\bar{V}^{-1}(\zeta, p)$, и, следовательно, функция $\Phi\left(\eta^{2}, \rho^{2} ; \bar{\theta} ; G\right)$ ведет себя в окрестности точек $p_{ \pm} \equiv \pm i \eta$, как $O\left(\rho^{-2 \mu}\right)$, при $\rho \rightarrow 0$, где $0<\mu<1$. Вследствие того же ограничения на самопересечения траектории функция $\Phi\left(\eta^{2}, \rho^{2} ; \bar{\theta} ; G\right)$ должна неограниченно возрастать и при $|\rho| \rightarrow \infty$. Из приведенных выше обсуждений предполагаемого поведения функции $\Phi\left(\eta^{2}, \rho^{2} ; \bar{\theta} ; G\right)$, а также из уравнений $(27)$ и (28) следует, что в качестве исходного приближения для искомой функции $\bar{V}(\zeta, p)$ можно принять функцию

$$
\mathcal{V}(\eta, p)=A \rho^{2(\mu-1)} \bar{K}_{1-\mu}(a \rho)
$$

в которой $\bar{K}_{\kappa}(z)=z^{\kappa} K_{\kappa}(z)$, а значения параметров $A, a$ и $\mu=\mu(d)$ при выполнении условий

$$
0<\mu<1, \quad 2 \leqslant d<4 \quad(0 \leqslant s<1)
$$

должны быть еще найдены. Параметр $a$ является некоторой характеристической длиной, пропорциональной величине $\sqrt{\left\langle r^{2}\right\rangle}$ с коэффициентом пропорциональности, зависящим от размерности пространства $\mathbb{R}^{d}$. Тогда согласно формулам (26) и (37) соответствующее приближение для функции $\widehat{V}(\eta, r)$ есть

$$
\widehat{\mathcal{V}}(\eta, r)=(2 \pi)^{-d / 2} A R^{-2 \nu} \bar{K}_{\nu}(\eta R),
$$

где

$$
R^{2} \equiv r^{2}+a^{2}, \quad \nu \equiv \mu+s, \quad s<\nu<1 .
$$

Ниже мы воспользуемся асимптотическим поведением функции $K_{\kappa}(z), \kappa \neq 1$, при малых и больших значениях аргумента $z$ :

$$
\begin{aligned}
\frac{2^{1-\kappa}}{\Gamma(\kappa)} \bar{K}_{\kappa}(z) & =1-\frac{\Gamma(1-\kappa)}{\Gamma(1+\kappa)}\left(\frac{z}{2}\right)^{2 \kappa}+\frac{1}{(1-\kappa)}\left(\frac{z}{2}\right)^{2}+O\left(z^{2(1+\kappa)}\right), \quad z \rightarrow 0, \\
K_{\kappa}(z) & =\sqrt{\frac{\pi}{2 z}} e^{-z}\left[1+O\left(z^{-1}\right)\right], \quad|z| \rightarrow \infty
\end{aligned}
$$


Кроме того, функция $K_{\kappa}(z)$ при каждом фиксированном значении $z \neq 0$ является целой функцией от индекса $\kappa$, который здесь связан с величиной $d$. Для того чтобы найти значение параметра $\mu$, воспользуемся следующим способом. В соответствии с формулой (25), исходным приближением (37) и его следствием (38) рассмотрим для плотности вероятности $W_{n}(\mathbf{r})$ приближение

$$
\mathcal{W}_{n}(\mathbf{r})=\left[\int_{\bar{\gamma}} \zeta^{n} \mathcal{V}(\eta, 0) d \zeta\right]^{-1} \int_{\bar{\gamma}} \zeta^{n} \widehat{\mathcal{V}}(\eta, \mathbf{r}) d \zeta
$$

для которого получим асимптотическую оценку, когда $n \rightarrow \infty$, но значение $r$ при этом остается фиксированным. Поскольку плотность $\mathcal{W}_{n}(\mathbf{r})$ - положительная величина и, следовательно,

$$
\operatorname{Im} \mathcal{W}_{n}(\mathbf{r})=0
$$

то выполнения этого условия потребуем и для ее асимптотики. Далее, в выражении (40) перейдем от переменной $\zeta$ к переменной $\eta$ с помощью формулы (24), имеющей теперь вид

$$
\zeta-1=z_{0}(\bar{w}(i \eta)-1)+F(\zeta, i \eta ; \bar{\theta} ; \mathcal{V})-F(1,0 ; \bar{\theta} ; \mathcal{V})
$$

где

$$
\begin{aligned}
F(\zeta, p ; \bar{\theta} ; \mathcal{V})=- & \int \frac{d \mathbf{p}_{1}}{(2 \pi)^{d}} \bar{\theta}\left(\mathbf{p}_{1}\right) \mathcal{V}\left(\eta, \mathbf{p}-\mathbf{p}_{1}\right)+ \\
& +\int \frac{d \mathbf{p}_{1}}{(2 \pi)^{d}} \int \frac{d \mathbf{p}_{2}}{(2 \pi)^{d}} \bar{\theta}\left(\mathbf{p}_{1}\right) \bar{\theta}\left(\mathbf{p}_{2}\right) \times \\
& \times \mathcal{V}\left(\eta, \mathbf{p}-\mathbf{p}_{1}\right) \mathcal{V}\left(\eta, \mathbf{p}-\mathbf{p}_{1}-\mathbf{p}_{2}\right) \mathcal{V}\left(\eta, \mathbf{p}-\mathbf{p}_{2}\right)-\cdots
\end{aligned}
$$

Для исследования сходимости ряда в (43) рассмотрим интеграл

$$
\mathcal{I}\left(\mathbf{p}, \mathbf{p}_{1}\right)=-\int \frac{d \mathbf{p}_{2}}{(2 \pi)^{d}} \bar{\theta}\left(\mathbf{p}_{2}\right) \mathcal{V}\left(\eta, \mathbf{p}-\mathbf{p}_{1}-\mathbf{p}_{2}\right) \mathcal{V}\left(\eta, \mathbf{p}-\mathbf{p}_{2}\right)
$$

реализующий переход от первого члена этого ряда к его второму члену, подобно интегралу (36). Для краткости оценим величину интеграла $\mathcal{I}(\mathbf{0}, \mathbf{0})$ при $\eta=0$, область интегрирования для которого мы ограничим малым объемом шара радиуса $\left|\mathbf{p}_{2}\right|=p_{0}$ с центром в точке $\mathbf{p}_{2}=\mathbf{0}$. В результате мы получим величину порядка $O\left(p_{0}^{4 \mu+2 s-2}\right)$ в случае $p_{0} \rightarrow 0$. Отсюда следует, что сходимость интеграла (44) на нижнем пределе обеспечивается выполнением одного из эквивалентных условий

$$
\mu>\frac{1-s}{2}, \quad \nu>\frac{1+s}{2}
$$

в силу равенства $\nu=\mu+s$, а сходимость его на верхнем пределе обусловлена экспоненциальным стремлением к нулю подынтегральной функции. Вычисление интегралов в (43) в окрестности точек $p=0$ и $\eta=0$ приводит к следующему результату:

$$
F(\zeta, p ; \bar{\theta} ; \mathcal{V})=\sigma(p)+\tau(p) \eta^{2 \nu}+O\left(a^{2} \eta^{2}\right)
$$


где

$$
\begin{gathered}
\sigma(p)=-A_{0} a^{-2 \nu} \bar{\theta}(p)+A_{1} a^{2(1+s-3 \nu)} \bar{\theta}^{2}(p) \bar{K}_{3 \nu-s-1}(a p)-\cdots, \\
\tau(p)=\frac{A_{0} \Gamma(1-\nu)}{4^{\nu} \Gamma(1+\nu)} \bar{\theta}(p)-A_{2} a^{2(1+s-2 \nu)} \bar{\theta}^{2}(p) \bar{K}_{2 \nu-s-1}(a p)+\cdots \\
A_{0}=c_{0} A, \quad A_{1}=c_{1} A^{3}, \quad A_{2}=c_{2} A^{3}
\end{gathered}
$$

$c_{0}, c_{1}, c_{2}$ суть определенные положительные числа.

Подставим теперь выражение (45) в (42) при малых значениях $\eta$, учитывая равенство $\bar{w}(i \eta)-\bar{w}(0)=O\left(\eta^{2}\left\langle r^{2}\right\rangle\right)$, а также соотношения

$$
\begin{aligned}
& \sigma(i \eta)-\sigma(0)=O\left(A^{3} v^{2} \eta^{2(3 \nu-s-1)}\right), \quad \eta \rightarrow 0, \\
& \tau(i \eta)-\tau(0)=O\left(A^{3} v^{2} \eta^{2(2 \nu-s-1)}\right), \quad \eta \rightarrow 0 .
\end{aligned}
$$

В результате мы получим искомую связь между переменными $\zeta$ и $\eta$ :

$$
\zeta-1=\tau_{0} \eta^{2 \nu}+O\left(A^{3} v^{2} \eta^{2(3 \nu-s-1)}\right), \quad \tau_{0}>0,
$$

где положительное значение величины $\tau_{0} \equiv \tau(0)$, как следует из указанной выше формулы для $\tau(p)$, обеспечивается соответствующим выбором числовой величины $A^{2} v a^{2(1+s-2 \nu)}$, в которой $1+s-2 \nu<0$.

Обратимся теперь к формуле (40) и выполним асимптотическую оценку плотности $\mathcal{W}_{n}(\mathbf{r})$, когда $n \rightarrow \infty$, но значение $r$ при этом остается фиксированным. С этой целью в интегралах формулы (40) удобно ввести новую переменную интегрирования $\xi$ посредством равенства $\zeta-1=\tau_{0} a^{-2 \nu} \xi$, вследствие которого из (46) следует

$$
\xi=(a \eta)^{2 \nu}+O\left(A^{3} v^{2} \eta^{2(3 \nu-s-1)}\right), \quad \eta \rightarrow 0,
$$

а контур интегрирования $\bar{\gamma}$ в (40) по этой переменной тогда будет расположен в ее правой полуплоскости. Теперь проведем разрез в комплексной плоскости переменной $\xi$ вдоль ее отрицательной вещественной оси и в обоих интегралах формулы (40) сдвинем контур интегрирования $\bar{\gamma}$ бесконечно далеко в левую полуплоскость, обходя особые точки подынтегральных функций, среди которых точка $\xi=0$ имеет наибольшую вещественную часть. Поэтому наибольший вклад в величину $\mathcal{W}_{n}(\mathbf{r})$ при $n \rightarrow \infty$ вносит результат интегрирования в (40) по той части контура $\bar{\gamma}$, которая охватывает указанный разрез $(-\infty, 0]$. Далее, если обозначить через $\xi_{-}=\xi e^{-i \pi}$ и $\xi_{+}=\xi e^{i \pi}$, где $\xi>0$, значения переменной $\xi$ соответственно на нижнем и на верхнем берегах разреза $(-\infty, 0]$ и в формуле $(40)$ учесть результат интегрирования лишь по контуру, охватывающему этот разрез, получим

$$
\begin{aligned}
\mathcal{W}_{n}(\mathbf{r}) \sim & {\left[\int_{0}^{\infty} e^{-n \tau_{0} a^{-2 \nu} \xi}\left(\mathcal{V}\left(\eta_{+}, 0\right)-\mathcal{V}\left(\eta_{-}, 0\right)\right) d \xi\right]^{-1} \times } \\
& \times \int_{0}^{\infty} e^{-n \tau_{0} a^{-2 \nu} \xi}\left(\widehat{\mathcal{V}}\left(\eta_{+}, r\right)-\widehat{\mathcal{V}}\left(\eta_{-}, r\right)\right) d \xi, \quad n \rightarrow \infty
\end{aligned}
$$

а переменные $\eta_{-}=\eta_{-}(\xi)$ и $\eta_{+}=\eta_{+}(\xi)$ согласно (47) связаны с $\xi$ вблизи точки $\xi=0$ на нижнем и верхнем берегах разреза $(-\infty, 0]$ как

$$
\begin{aligned}
\xi e^{-i \pi} & =\left(a \eta_{-}\right)^{2 \nu}+O\left(\left(a \eta_{-}\right)^{2(3 \nu-s-1)}\right), & & \eta_{-} \rightarrow 0, \\
\xi e^{i \pi} & =\left(a \eta_{+}\right)^{2 \nu}+O\left(\left(a \eta_{+}\right)^{2(3 \nu-s-1)}\right), & & \eta_{+} \rightarrow 0 .
\end{aligned}
$$


Асимптотическая оценка интегралов в (48) при $n \rightarrow \infty$ дает

$$
\mathcal{W}_{n}(\mathbf{r}) \sim C_{0} \frac{\left(r^{2}+a^{2}\right)^{1-\nu}}{\left(\tau_{0} n\right)^{(2-\mu) / \nu}}\left[\frac{e^{i \pi / \nu}-e^{-i \pi / \nu}}{e^{i \pi(\mu-1) / \nu}-e^{-i \pi(\mu-1) / \nu}}\right],
$$

где $C_{0}$ - определенная положительная постоянная. Согласно условию (41) величина, фигурирующая в квадратных скобках асимптотического выражения (49), должна быть положительной. Последнее требование соблюдается при выполнении равенства

$$
\frac{\pi}{\nu}+\frac{\pi}{\nu}(1-\mu)=2 \pi k, \quad k=1,2, \ldots,
$$

которое совместимо с условием $\nu=\mu+s$ лишь для $k=1$. Отсюда находим

$$
\mu=\frac{2}{3}(1-s)=\frac{4-d}{3}, \quad \nu=\frac{s+2}{3}=\frac{d+2}{6} .
$$

Таким образом, искомая асимптотика плотности $\mathcal{W}_{n}(\mathbf{r})$ при $n \rightarrow \infty$ имеет следующий вид:

$$
\mathcal{W}_{n}(\mathbf{r}) \sim C_{0}\left(r^{2}+a^{2}\right)^{(1-s) / 3}\left(\tau_{0} n\right)^{-2} .
$$

Здесь следует отметить, что отличие поведения последнего выражения от асимптотики плотности нормального распределения при $n \rightarrow \infty$ становится менее существенным, когда размерность $d$ растет, приближаясь к $d=4$. Этот вывод можно объяснить ослаблением эффекта, обусловленного запретом самопересечений траектории, при увеличении пространственной размерности. Теперь в соответствии с приближением (37) для функции $\bar{V}(\zeta, p)$ определим приближение $\bar{G}$ для величины $G$ аналогично равенству (27):

$$
\mathcal{V}(\eta, p)=\bar{V}_{0}(\eta, p) \bar{G},
$$

и проверим будет ли функция $\bar{G}$ удовлетворять в определенном смысле уравнению

$$
\bar{G}^{-1}=1+\Phi\left(\eta^{2}, \rho^{2} ; \bar{\theta} ; \bar{G}\right),
$$

аналогичному уравнению (28).

Эту проверку мы проведем при $\eta=0$ и в окрестности точки $p=0$, где по предположению находится точка нормировки $p^{2}=\lambda$. С этой целью обратимся к формулам (29) и (43) и вычислим асимптотику функции

$$
\Phi\left(0, p^{2} ; \bar{\theta} ; \bar{G}\right)=\bar{V}_{0}(0, p)[F(1,0 ; \bar{\theta} ; \mathcal{V})-F(1, p ; \bar{\theta} ; \mathcal{V})]
$$

при $p^{2} \rightarrow 0$. Подставляя в правую часть $(51)$ выражение для $F(\zeta, p ; \bar{\theta} ; \mathcal{V})$ из (45), приходим к равенству

$$
\Phi\left(0, p^{2} ; \bar{\theta} ; \bar{G}\right)=\bar{V}_{0}(0, p)[\sigma(0)-\sigma(p)]
$$

в котором

$$
\begin{gathered}
\sigma(0)-\sigma(p)=A_{0} a^{-2 \nu} v\left[\Lambda_{s+1}\left(r_{0} p\right)-1\right]+A_{1} a^{-2} v^{2}\left[\bar{K}_{1}(0)-\Lambda_{s+1}^{2}\left(r_{0} p\right) \bar{K}_{1}(a p)\right], \\
\Lambda_{s+1}(z)=1-\frac{z^{2}}{4(s+2)}+O\left(z^{4}\right), \quad z \rightarrow 0 \\
\bar{K}_{1}(z)=1+\frac{z^{2}}{2} \ln \frac{z}{2}+(2 \gamma-1)\left(\frac{z}{2}\right)^{2}+O\left(z^{4} \ln z\right), \quad z \rightarrow 0 .
\end{gathered}
$$


Учитывая в (51) приведенные выше значения функций $\Lambda_{s+1}(z)$ и $\bar{K}_{1}(z)$, мы приходим к следующему выражению:

$$
\Phi\left(0, p^{2} ; \bar{\theta} ; \bar{G}\right)=-\chi z_{0}^{-1} a^{-2} v^{2} A^{3} \ln \left(a^{2} p^{2}\right)+\Omega\left(A, a, r_{0}\right)+O\left(r_{0}^{2} p^{2} \ln \left(a^{2} p^{2}\right)\right),
$$

когда $p \rightarrow 0$, где не зависящая от $p$ величина $\Omega\left(A, a, r_{0}\right)$ связывает указанные параметры задачи, а $\chi$ - некоторое положительное число, зависящее от размерности пространства. Таким образом, с одной стороны, согласно определениям (37) и (50) в малой окрестности точки $p=0$ имеем

$$
\left.\frac{\partial \bar{G}(0, p)}{\partial \ln p^{2}}\right|_{p^{2}=\lambda}=\mu,
$$

а с другой стороны, функция $\bar{G}(0, p)$ должна удовлетворять уравнению

$$
\bar{G}^{-1}(0, p)=1+\Phi\left(0, p^{2} ; \bar{\theta} ; \bar{G}\right)
$$

в его дифференциальной форме

$$
\left.\frac{\partial \bar{G}(0, p)}{\partial \ln p^{2}}\right|_{p^{2}=\lambda}=-\left.\frac{\partial \Phi\left(0, p^{2} ; \bar{\theta} \bar{G}^{2} ; \bar{G}\right)}{\partial \ln p^{2}}\right|_{p^{2}=\lambda},
$$

согласно РГ-уравнению (34). Следовательно, правые части уравнений (52) и (54) должны совпадать, чтобы выражение (37) с найденными значениями $\mu$ и $\nu$ можно было рассматривать в качестве исходного приближения для решения основного уравнения. Отсюда находим, что

$$
A=\mathcal{C}(d)\left[z_{0}\left\langle r^{2}\right\rangle v^{-2}\right]^{1 / 3}
$$

где $\mathcal{C}(d)$ - положительное число, зависящее от размерности пространства $\mathbb{R}^{d}$.

Теперь мы можем установить явный вид функций $\mathcal{V}(\eta, 0)$ и $\widehat{\mathcal{V}}(\eta, \mathbf{r})$ и, следовательно, найти с помощью равенства (40) асимптотику плотности вероятности. Последнюю мы определим с помощью метода перевала, когда $(r / a) \rightarrow \infty$ и $n \rightarrow \infty$, но отношение $(r / n a)$ фиксировано и мало. В результате для главного члена асимптотического разложения плотности $\mathcal{W}_{n}(\mathbf{r})$ при указанных выше условиях имеем

$$
\mathcal{W}_{n}(\mathbf{r}) \sim C_{d}\langle R\rangle^{-d} Z^{(4-d) D / 6} e^{-c_{d} Z^{D}}
$$

где

$$
\langle R\rangle=\left(\tau_{0} n\right)^{3 /(d+2)}, \quad Z \equiv \frac{R}{\langle R\rangle}=\frac{\sqrt{r^{2}+a^{2}}}{\left(\tau_{0} n\right)^{3 /(d+2)}}, \quad D=\frac{d+2}{d-1},
$$

a $c_{d}$ и $C_{d}$ - вполне определенные положительные числа [10].

Случай $d=4$ особый и его следует рассматривать отдельно. Тем не менее полученный результат позволяет нам предположить, что в случае $d>4$ асимптотика плотности вероятности при рассмотренных выше условиях должна иметь форму нормального распределения [11], [12]. Это означает, что в этом случае запрет самопересечений траектории оказывает несущественное влияние на распределение расстояния между концами траектории блуждающей частицы. 


\section{4. КАНОНИЧЕСКИЙ АНСАМБЛЬ САМОИЗБЕГАЮЩИХ БЛУЖДАНИЙ}

Обратимся к формулам (25) и (26), связывающим плотность вероятности $W_{n}(\mathbf{r})$ с функцией $\bar{V}(\zeta, \mathbf{p})$, для которой получено основное уравнение в рассматриваемой задаче. Введем обозначения

$$
\bar{Q}_{n} \equiv \int_{\bar{\gamma}} d \zeta \zeta^{n} \bar{V}(\zeta, 0), \quad \widetilde{Q}(\zeta) \equiv \sum_{n=0}^{\infty} \bar{Q}_{n}^{-1} \zeta^{n},
$$

тогда согласно равенству (3) мы получим

$$
\mathrm{E} W_{n}(\mathbf{r})=\left(q^{-1}-1\right) \int_{\bar{\gamma}} d \zeta \widetilde{Q}(\zeta) \widehat{V}\left(q^{-1} \zeta, \mathbf{r}\right), \quad q^{-1}=1+N^{-1} .
$$

Аналогично изложенному выше подходу запишем исходное приближение (40) посредством равенства

$$
\mathcal{W}_{n}(\mathbf{r})=U_{n}^{-1} \int_{\bar{\gamma}} d \zeta \zeta^{n} \widehat{\mathcal{V}}(\eta, \mathbf{r})
$$

в котором

$$
U_{n} \equiv \int_{\bar{\gamma}} d \zeta \zeta^{n} \mathcal{V}(\eta, 0)
$$

а связь между переменными $\zeta$ и $\eta$ определена в (46). Теперь усредним указанное выражение для $\mathcal{W}_{n}(\mathbf{r})$ по распределению (2):

$$
\mathrm{E} \mathcal{W}_{n}(\mathbf{r})=(1-q) \int_{\bar{\gamma}} d \zeta \widetilde{U}(q \zeta) \widehat{\mathcal{V}}(\eta, \mathbf{r})
$$

где

$$
\widetilde{U}(\zeta) \equiv \sum_{n=0}^{\infty} U_{n}^{-1} \zeta^{n}
$$

Однако из равенства (55) с помощью формулы обращения имеем

$$
\sum_{n=0}^{\infty} z^{n} U_{n}=\frac{2 \pi}{i z} \mathcal{V}\left(\left(\frac{1-z}{\tau_{0} z}\right)^{1 / 2 \nu}, 0\right)
$$

откуда получим, что согласно выражению (37) асимптотическое поведение суммы в последнем равенстве описывается следующим образом:

$$
\sum_{n=0}^{\infty} z^{n} U_{n} \sim \frac{\bar{A}}{i}\left(\frac{\tau_{0}}{1-z}\right)^{(1+2 s) /(2+s)}, \quad z \rightarrow 1-0,
$$

где $\bar{A}=2 \pi A \bar{K}_{1-\mu}(0)$. Тогда тауберова теорема утверждает, что

$$
U_{n} \sim \frac{\bar{A}}{i \Gamma((1+2 s) /(2+s))} \tau_{0}^{(1+2 s) /(2+s)} n^{-(1-s) /(2+s)}, \quad n \rightarrow \infty,
$$


вследствие чего ряд, представляющий функцию $\widetilde{U}(q \zeta)$ под знаком интеграла в $(56)$, сходится при условии $0 \leqslant|\zeta|<q^{-1}$. Так как $U_{n}^{-1}$ суть коэффициенты ряда, представляющего функцию $\widetilde{U}(z)$, то, используя тауберову теорему в обратном порядке, получим

$$
\begin{aligned}
\widetilde{U}\left(\frac{\zeta}{\zeta_{0}}\right) \sim \frac{i}{\bar{A}} \Gamma\left(\frac{3}{2+s}\right) \Gamma & \left(\frac{1+2 s}{2+s}\right) \tau_{0}^{-(1+2 s) /(2+s)}\left(\frac{\zeta_{0}}{\zeta_{0}-\zeta}\right)^{3 /(2+s)}, \\
& \zeta \rightarrow \zeta_{0} \equiv q^{-1}
\end{aligned}
$$

Поскольку наибольший интерес здесь представляет поведение функции Е $\mathcal{W}_{n}(\mathbf{r})$ для больших значений $r / a$ и $N$, то в силу свойств функции $\widehat{\mathcal{V}}(\eta, \mathbf{r})$ главный вклад в величину интеграла в $(56)$ вносит область интегрирования с малыми значениями $\left|\zeta_{0}-\zeta\right|$ или $\left|N^{-1}-\tau_{0} \eta^{2 \nu}\right|$ согласно (46). Поэтому для оценки интеграла в (56) при $r / a \gg 1$ и $N \gg 1$ мы воспользуемся асимптотическим выражением функции $\widetilde{U}\left(\zeta / \zeta_{0}\right)$ в (57) и запишем формулу (56) в виде

$$
\mathrm{EW}_{n}(\mathbf{r}) \sim \frac{\nu \mathcal{C}}{i(1-s) N R^{2 \nu} \tau_{0}^{(1+2 s) /(2+s)}} \frac{d}{d \zeta_{0}} \int_{\bar{\gamma}} d \zeta\left(\zeta_{0}-\zeta\right)^{-(1-s) /(2+s)} \bar{K}_{\nu}(\eta R),
$$

где

$$
\mathcal{C}=\frac{3}{(2 \pi)^{s+2} \bar{K}_{1-\mu}(0)} \Gamma\left(\frac{3}{2+s}\right) \Gamma\left(\frac{1+2 s}{2+s}\right) .
$$

Для оценки интеграла в (58) проведем разрез в комплексной плоскости переменной $\zeta$ по лучу $\left[\zeta_{0}, \infty\right)$ и сдвинем контур интегрирования $\bar{\gamma}$ бесконечно далеко в правую полуплоскость. Тогда, учитывая экспоненциальный характер стремления к нулю подынтегральной функции при $\zeta \rightarrow+\infty$, мы получим после интегрирования по указанному контуру искомый результат:

$$
\mathrm{EW}_{n}(\mathbf{r}) \sim \frac{\mathcal{C}}{1-s} \sin \left(\frac{1-s}{2+s} \pi\right)\left(\tau_{0} N\right)^{-d / 2 \nu} J\left(\frac{R}{\left(\tau_{0} N\right)^{1 / 2 \nu}}\right)
$$

- асимптотику плотности вероятности расстояния $r$ между концами траектории частиц канонического ансамбля, блуждающих без самопересечений в $\mathbb{R}^{d}$, где

$$
J(x)=\int_{0}^{\infty} d t t^{-(1-s) /(2+s)} \bar{K}_{1-\nu}\left(x(1+t)^{1 / 2 \nu}\right)
$$

- монотонно убывающая функция в области $(0, \infty)$.

\section{Список литературы}

[1] К. Хуанг, Статистическая механика, Мир, М., 1966.

[2] М. В. Федорюк, Метод перевала, Наука, М., 1977.

[3] В.И. Алхимов, " $d$-Мерная модель канонического ансамбля открытых струн”, ТМФ, 180:1 (2014), 125-144.

[4] В.И. Алхимов, “Эффект исключенного объема в статистике самоизбегающих блужданий”, УФН, 164:6 (1994), 561-601.

[5] Е. Титчмарш, Теория функиий, Наука, М., 1980.

[6] В. Феллер, Введение в теорию вероятностей и ее приложения, т. 2, Мир, М., 1967. 
[7] Н. Н. Боголюбов, Д. В. Ширков, Введение в теорию квантованных полей, Наука, М., 1976.

[8] Л. В. Овсянников, Групповой анализ дифференииалъных уравнений, Наука, М., 1978.

[9] M. Gell-Mann, F. E. Low, "Quantum electrodynamics at small distances", Phys. Rev., 95:5 (1954), 1300-1312.

[10] V.I. Alkhimov, "Self-avoiding random walk in $d<4$ dimensions", Phys. Lett. A, 133:1-2 (1988), 15-17.

[11] D. Bridges, T. Spencer, "Self-avoiding walk in 5 or more dimensions", Commun. Math. Phys., 97:1 (1985), 125-148.

[12] T. Hara, G. Slade, "The lace expansion for self-avoiding walk in five or more dimensions", Rev. Math. Phys., 4:2 (1992), 235-327.

Поступила в редакцию 25.01.2016, после доработки 14.02.2016 